\title{
BMJ Open Changes in health-related quality of life (HRQoL) after discharge from intensive care unit: a protocol for a systematic review
}

\author{
Alice M J Gerth, ${ }^{1}$ Peter J Watkinson, ${ }^{2}$ J Duncan Young $^{3}$
}

To cite: Gerth AMJ, Watkinson PJ, Young JD. Changes in health-related quality of life (HRQOL) after discharge from intensive care unit: a protocol for a systematic review. BMJ Open 2015;5:e009508.

doi:10.1136/bmjopen-2015009508

\section{- Prepublication history} and additional material is available. To view please visit the journal (http://dx.doi.org/ 10.1136/bmjopen-2015009508).

Received 23 July 2015 Revised 4 September 2015 Accepted 14 September 2015

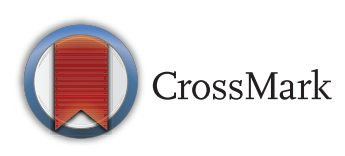

${ }^{1}$ Kadoorie Centre for Critical Care Research and Education, John Radcliffe Hospital, Oxford, UK

${ }^{2}$ Kadoorie Centre for Critical Care and Trauma Research and Education, John Radcliffe Hospital, Oxford, UK ${ }^{3}$ Nuffield Department of Clinical Neurosciences, University of Oxford, Oxford, UK

Correspondence to Dr Alice M J Gerth; alice.gerth@medsci.ox.ac.uk

\section{ABSTRACT}

Introduction: Treatment on an intensive care unit (ICU) imposes a high treatment burden on patients, as well as an economic burden for the healthcare provider. Many studies have recorded health-related quality of life (HRQoL) in patients after treatment on an ICU. We propose a systematic review of these studies.

Methods: We will search the National Library of Medicine's PubMed electronic database (PubMed), the Cochrane database, Cumulative Index to Nursing and Allied Health Literature (CINAHL), Web of Science and Open Grey to identify papers reporting quality of life after discharge from ICU. We will include papers including validated quality of life measures. We will examine three categories: populations of patients treated on general ICUs, patients with severe infections and patients with respiratory dysfunction. We will extract HRQoL data. We will assess papers for risk of bias using the QUADAS-2 tool. The strength of our conclusions will depend on the quality and number of papers showing uniform results.

Ethics and dissemination: This review will use published literature and contains no primary data; so we do not need ethical approval. We will submit the outcome of the systematic review to a peer-reviewed journal.

Trial registration number: PROSPERO CRD42015024700.

\section{INTRODUCTION}

\section{Rationale}

Treatment on an intensive care unit (ICU) imposes a high treatment burden on patients, as well as an economic burden for the healthcare provider. Currently we know much about survival through the Intensive Care National Audit and Research Centre (ICNARC). ICNARC collates data on ICU admission and mortality. ICNARC recorded approximately 142038 admissions to 202 ICUs in England, Wales and Northern Ireland from 1 April 2013 to 31 March 2014. Of these approximately $80 \%$ survived to hospital discharge. ${ }^{1}$ We know less about quality of life after discharge than survival. For

\section{Strengths and limitations of this study}

- We will focus on the general effect of intensive care unit rather than the conditions that cause admission.

- The review will only include those with a high inclusion rate so that the results are generalisable and will only include studies using validated quality of life measures.

- Recent studies have longer follow-ups and so we hope to see whether the improvement in quality of life continues or reaches a plateau.

- This study is limited by not being suitable for a meta-analysis.

patients their quality of life after ICU is very important and informs whether they believe admission to ICU is or was in their best interests. There has been an increase in research over the past 15 years in quality of life after ICU that we will analyse and summarise in this systematic review.

This review will identify studies where validated measures of health-related quality of life (HRQoL) were determined after treatment in an ICU, and the time the measurements were taken. This will allow us to assess the HRQoL compared to the general population, how HRQoL changes with time after ICU discharge and variables affecting HRQoL. Other areas of interest include subdomains of HRQoL (especially the difference between mental health recovery and physical health recovery); method of HRQoL measurement; the length of follow-up and the loss of patients to follow-up over time.

It will also inform future research by identifying gaps in our knowledge or areas lacking clarity.

\section{Objectives}

A systematic review of all studies reporting the HRQoL for patients discharged alive 
after treatment in an ICU. To answer the primary question 'What affect does admission to an ICU have on quality of life for all patients discharged alive compared to an age and gender-matched general population?' A reliable understanding of quality of life after discharge will help inform future healthcare decisions and economics. Secondary questions include: 'Does quality of life improve with time after discharge?' 'How are quality of life studies undertaken with regard to follow-up duration, quality of life measures used and successful follow-up rates?'

\section{METHODS AND ANALYSIS \\ Design}

This protocol is based on the PRISMA-P guidelines for systematic reviews. ${ }^{2}$ We will carry out a systematic review of literature published in peer reviewed journals on HRQoL after discharge from an ICU.

\section{Search methods}

We will search the National Library of Medicine's PubMed electronic database (PubMed), the Cochrane database, the Cumulative Index to Nursing and Allied Health Literature (CINAHL), Web of Science and Open Grey to identify papers reporting quality of life after discharge from ICU in all languages covering January 2000 to May 2015 (papers pre 2000 are unlikely to reflect current ICU admissions and care). We selected the search terms with advice from a medical librarian. They are broad to capture all potential studies. Our search terms are: ('quality of life' OR 'qaly' OR 'health related quality of life)' AND ('intensive care' OR 'icu' OR 'critical care' OR 'itu' OR 'intensive treatment unit' OR 'intensive therapy unit'). We will search title, abstracts and key words.
We will screen the reference lists of included studies and published literature reviews for further eligible studies. Studies identified in this manner will be examined for why the initial search terms had not identified them. If need be we will search with a new 'key word'.

\section{Types of study to be included}

We will include prospective cohort studies, retrospective cohort studies, case-control studies, cross-sectional studies and randomised controlled trials. In the case of interventional studies the data will be taken from the control arm.

\section{Data management}

A reference manager programme will be used to store identified citations and their electronic text.

\section{Study selection}

Studies will go through three levels of selection: title, abstract and full text (figure 1). After the PubMed search, one reviewer will screen the titles to identify potentially relevant studies. We will collect the abstracts of these studies. Two researchers will remove abstracts that clearly do not match the eligibility criteria (recording the reason why).

Two researchers will carry out a final screening process of the full-text articles to select articles that reach all our eligibility criteria. Again we will record the reason for exclusion. It is likely that at this point we will divide the studies into three groups. (1) Studies reporting an unselected patient population. (2) Studies reporting patients admitted with severe infections. (3) Studies reporting patients admitted with respiratory compromise (including prolonged mechanical ventilation, acute respiratory distress syndrome and acute lung injury).
Figure 1 Sample flow diagram, adapted from PRISMA statement 2009. ${ }^{3}$

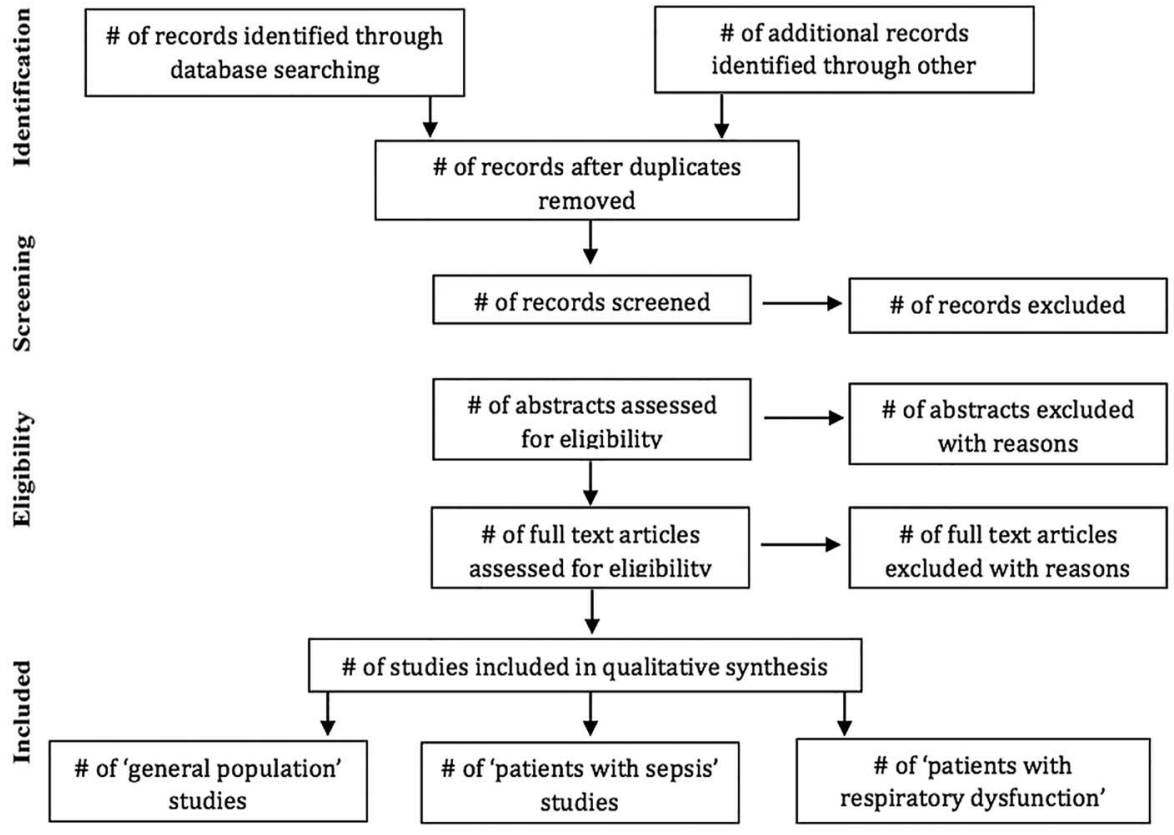


The inclusion of papers in each stage of the systematic review will be shown in a diagram adapted from the PRISMA statement ${ }^{3}$ (see figure 1).

\section{Eligibility criteria}

We will use the following eligibility criteria so that the data represents a global adult ICU population or the two subpopulations of interest. We will only use validated quality of life measures and set time points of data collection. This will allow comparison between papers.

Inclusion criteria

- Participants 16 years or older

- Original research

- Quality of life assessed at least at 3 months

- General ICUs (medical/surgical/mixed)

- Specific time points of data collection $( \pm 1$ week if $<12$ months, \pm 1 month if $>12$ months)

- Quality of life assessed using any validated HRQoL scale including:

- Quality of Well Being Scale ${ }^{4}$

- EuroQoL ${ }^{5}$

- Nottingham Health Profile ${ }^{6}$

- Short Form 36/12 8

- Sickness Impact Profile ${ }^{9}$

- Health Utilities Index ${ }^{10}$

Exclusion criteria

- Population investigated specific to one disease or procedure or event, for example, post cardiac arrest

- Population investigated with an age-specific lower boundary, for example, $>65$ years

- Population investigated specific to outcome, for example, only looking at those who describe their outcome as poor/good.

- Reporting on same patient data set as another study.

- In these cases we will include the study with longest follow-up. If follow-up duration is the same we will choose those with the largest initial patient cohort.

- Interventional study with no control group.

\section{Data extraction}

One researcher will be responsible for data extraction and a second will be consulted if data is unclear, if a consensus cannot be reached it will not be included. We will populate a piloted pre-specified data extraction table. We will extract data from the full-text article and any online supplementary data. Where there is lack of clarity in the data, we will contact the authors, if this is unsuccessful, we will exclude ambiguous data.

We will extract the following information:

1. Citation

2. Study country of origin

3. Study design

4. Year of publication

5. Type of ICU

6. Patient type: general/sepsis/respiratory (Note. Definition as used by the author. Where definitions differ we will consider analysis by subgroup.)

7. Definition of sepsis
8. Study inclusion and exclusion criteria

9. Number of patients screened

10. Number of patients excluded

11. Number of patients alive at start of follow-up period (in appropriate category, eg, in control group if an interventional study)

12. Study group characteristics

A. Mean age of study group

B. Per cent male of study group

C. Per cent admitted for surgical reasons (to determine medical/surgical split of the ICU)

D. Mean ICU length of stay

E. Mean hospital length of stay

F. Any subgroups defined by length of stay

13. Follow-up time points

14. Mortality rate*

15. Lost to follow-up*

16. Description of those lost to follow-up

17. Successfully followed up*

18. Method of assessing quality of life pre. ICU if used

19. HRQoL tools used

20. HRQoL data, from paper and additional material online*

21. Method of assessment, for example, telephone, face to face

22. Involvement of proxies, for example, family
A. None
B. Assisted with answering questionnaires
C. Answered on patients behalf
D. Not specified/mixture of the above options

23. HRQoL relative to age and sex-adjusted normal population

24. Previous health/pre-existing disease

25. Trend in HRQoL postdischarge (improved, worsened etc)

26. Associations between HRQoL and other variables

27. Competing interests declared by authors

*At each follow-up time point.

We will record HRQoL scores for the broadest population. If the HRQoL is only reported by subgroup we will combine it (if number, mean and SD are available). If we cannot combine it we may include it as a subgroup of interest (patients with sepsis or respiratory dysfunction) or exclude it. We will record data in maximum detail, for example, for each domain of the HRQoL system used if available.

\section{DATA ANALYSIS}

\section{Synthesis of data and data analysis}

For each subgroup (general/sepsis/respiratory) and overall, we will describe quality of life after ICU in a narrative manner (and tabular if appropriate). Where possible, we will include comparisons with preceding quality of life assessments. We will describe secondary outcomes (length of follow-up, follow-up rates and mortality rates) in a narrative and tabular/graphical form (eg, graph of follow-up rates against time). 


\section{Assessment of bias and strength of conclusions}

Given the heterogeneity of the likely study designs and outcome measures, we do not plan to carry out a meta-analysis of the study results or statistically assess meta-biases. We will assess the papers for applicability and bias using the QUADAS-2 tool ${ }^{11}$ as well as study size and declarations of competing interests. The strength of our conclusions will depend on the quantity and uniformity of papers support a result and the spread between them. If we have sufficient studies we may analyse the effect of time on quality of life and successful follow-up rates.

\section{Assessment of strength of conclusions}

The strength of conclusions will depend on the quantity and uniformity of results and the spread between them.

\section{STRENGTHS AND LIMITATIONS}

\section{Why is a new review needed?}

We will build on previous reviews. ${ }^{12-15}$ Elliott's and Chaboyer's reviews in 1999 and 2000, reported on papers that are now over 15 years. At this point quality of life was a new part of ICU outcome research, many studies used their own (non-validated) measures and difficult to compare. ${ }^{12} 13$ They reported reduced quality of life in patients treated in an ICU after discharge compared to the general population and the challenge of patient retention. Adamson et al identified 34 papers published before 2003, of these 19 used validated HRQoL measures and only 3 looked at multiple time points. ${ }^{14}$ Dowdy et $a l \mathrm{~s}^{16}$ review from 2005 reported 27 studies with the most recent also being in 2003.This reported studies using validated quality of life measures. The major findings were that quality of life after discharge is less than the general public. There was a small improvement in quality of life over time and that a worse quality of life was seen in older patients with more severe illnesses. Oeyen et $a l^{15}$ reported on papers between 1999 and 2009 grouped by diagnostic category. They also reported that quality of life after ICU was less good than the general population with a slight improvement in physical health but not mental over time. This review will build on the previous ones. We will look at the past 15 years of studies. We will focus on the general effect of ICU rather than the conditions that cause admission. The review will only include those with a high inclusion rate so that the results are generalisable and will only include studies using validated quality of life measures. Recent studies have longer follow-ups and so we hope to see whether the improvement in quality of life continues or reaches a plateau.

This study is limited by not being suitable for a meta-analysis. We intend to use the identified knowledge gaps to inform future research.

\section{Dissemination plans}

The review will be submitted to a peer reviewed journal in healthcare.
Twitter Follow Alice Gerth at @amjgerth

Acknowledgements This work will be supported by the NIHR Oxford Biomedical Research Centre, Oxford University Hospitals NHS Trust, the Oxford Biomedical Research Centre and the Oxford University Clinical Academic Graduate School.

Contributors AMJG, PJW and JDY conceived the original idea. AMJG prepared the manuscript with revisions from JDY and PJW. All authors read and approved the final manuscript. JDY is the guarantor.

Funding NIHR Oxford Biomedical Research Centre, Oxford University Hospitals NHS Trust, the Oxford Biomedical Research Centre and the Oxford University Clinical Academic Graduate School support salaries to enable this work.

Competing interests None declared.

Provenance and peer review Not commissioned; externally peer reviewed.

Open Access This is an Open Access article distributed in accordance with the Creative Commons Attribution Non Commercial (CC BY-NC 4.0) license, which permits others to distribute, remix, adapt, build upon this work noncommercially, and license their derivative works on different terms, provided the original work is properly cited and the use is non-commercial. See: http:// creativecommons.org/licenses/by-nc/4.0/

\section{REFERENCES}

1. Intensive Care National Audit and Research Centre. Key statistics from the Case Mix Programme 1st April 2013 to 31st March 2014. Intensive Care National Audit and Research Centre. 2015. https:// www.icnarc.org/Our-Audit/Audits/Cmp/Reports/Summary-Statistics (accessed 4 Jun 2015).

2. Moher D, Shamseer L, Clarke M, et al., PRISMA-P Group. Preferred reporting items for systematic review and meta-analysis protocols (PRISMA-P) 2015 statement. Syst Rev 2015;4:1.

3. Moher D, Liberati A, Tetzlaff J, et al. Preferred reporting items for systematic reviews and meta-analyses: the PRISMA statement. PLOS Med 2009;6:e1000097.

4. Kaplan R, Anderson J, Ganiats T. The quality of well-being scale: rationale for a single quality of life index. In: Walker S, Rosser R, eds. Quality of life assessment: key issues in the 1990s SE-3. Springer, 1993:65-94.

5. Brooks R. EuroQol: the current state of play. Health Policy 1996;37:53-72.

6. Hunt SM, McEwen J, McKenna SP. Measuring health status: a new tool for clinicians and epidemiologists. J R Coll Gen Pract 1985;35:185-8.

7. Ware JE, Sherbourne CD. The MOS 36-item short-form health survey (SF-36). I. Conceptual framework and item selection. Med Care 1992;30:473-83.

8. Ware J, Kosinski M, Keller SD. A 12-Item Short-Form Health Survey: construction of scales and preliminary tests of reliability and validity. Med Care 1996;34:220-33.

9. De Bruin AF, de Witte LP, Stevens F, et al. Sickness impact profile: the state of the art of a generic functional status measure. Soc Sci Med 1992;35:1003-14.

10. Feeny D, Furlong W, Boyle M, et al. Multi-attribute health status classification systems. Health Utilities Index. Pharmacoeconomics 1995;7:490-502.

11. Whiting PF, Rutjes AWS, Westwood ME, et al. QUADAS-2 a revised tool for the quality assessment of diagnostic accuracy studies. Ann Intern Med 2011;155:529-36.

12. Chaboyer W, Elliott D. Health-related quality of life of ICU survivors: review of the literature. Intensive Crit Care Nurs 2000;16:88-97.

13. Elliott D. Measuring the health outcomes of general ICU patients: a systematic review of methods and findings. Aust Crit Care 1999;12:132-40.

14. Adamson H, Elliott D. Quality of life after a critical illness: a review of general ICU studies 1998-2003. Aust Crit Care 2005;18:50.

15. Oeyen SG, Vandijck DM, Benoit DD, et al. Quality of life after intensive care: a systematic review of the literature. Crit Care Med 2010;38:2386-400.

16. Dowdy DW, Eid MP, Sedrakyan A, et al. Quality of life in adult survivors of critical illness: a systematic review of the literature. Intensive Care Med 2005;31:611-20. 